\title{
Circuit Requirements of A Direct Conversion Paging Receiver
}

\author{
Zhiheng Chen, Student Member, IEEE, and Jack Lau, Member, IEEE
}

\begin{abstract}
Circuit requirements of a direct conversion FLEX paging receiver with the zero-crossing interpolation demodulator are derived by means of high level simulation. Impacts of the limitations on noise and nonlinearity characteristics of the RF stage, the in- and quadrature-phase mismatch, and the LO frequency drift are analyzed relative to the degradation of the bit error rate. Official specifications on radio paging receivers are introduced to ensure the minimum performance. It is shown that an RF front-end with $25 \mathrm{~dB}$ gain, $5.3 \mathrm{~dB}$ noise figure and $11 \mathrm{dBm}$ second order intercept point makes the receiver qualified for the sensitivity, desensitization and intermodulation requirements.
\end{abstract}

\section{INTRODUCTION}

$\mathrm{F}$ OR years, traditional pagers in many countries have been governed by a standard known as POCSAG which is based on a binary frequency shift keying (FSK) modulation scheme and runs at 512, 1200 or 2400 bps. The insatiable quest for information and the development of new paging markets in rapidly developing countries have called for a more efficient and higher speed system. One such system, FLEX, makes use of the 4-level FSK and runs at a top speed of $6400 \mathrm{bps}$. Countries such as China are steadily embracing such new system.

While the new system is more efficient, it imposes some RF design challenges. In the POCSAG system, over the years, designers have successfully converted the early double-conversion architecture to single-conversion to the low cost direct conversion architecture [1][2]. Direct conversion is very appealing particularly in the pager units because of its inherent simplicity which requires only one major IC for the RF section.

Simplification of the FLEX receiver can be similarly appreciated. Two of the major difficulties, namely the cancellation of the DC offset and the demodulation of the zeroIF 4-FSK signal, have been studied in a previous work[3]. The studies have discussed the feasibility of employing a harmonic mixer to suppress the self-mixing related DC offset to near noise level, and the use of zero-crossing interpolation (ZCI) technique in the demodulation. The ZCI technique helps enhance the sensitivity by increasing the number of zero-crossings in the I and Q channels[4].

In this paper, we study the circuit implications, from the low noise amplifier to the mixer to base-band demodulator, should the ZCI be used. Issues such as gain, nonlinearity and noise of the front-end blocks will be discussed. We will also reveal requirements of the LO. The official standards imposed by the Hong Kong Office of Telecommu-

The authors are with the Department of Electrical and Electronic Engineering, The Hong Kong University of Science \& Technology, Clear Water Bay, Kowloon, Hong Kong. Email: eeczh@ee.ust.hk and eejlau@ee.ust.hk. nications Authority (HKOFTA) and the Ministry of Post and Telecommunications of China on paging receivers are strictly observed. These are typical standards and should be consistent with most part of the world.

Section II describes the receiver models as well as the official specifications. The simulation results are discussed and compared to the specifications in section III. Requirements of the RF front-end circuits are then derived in section IV.

\section{Receiver Modeling and Specifications}

\section{A. The Receiver Model}

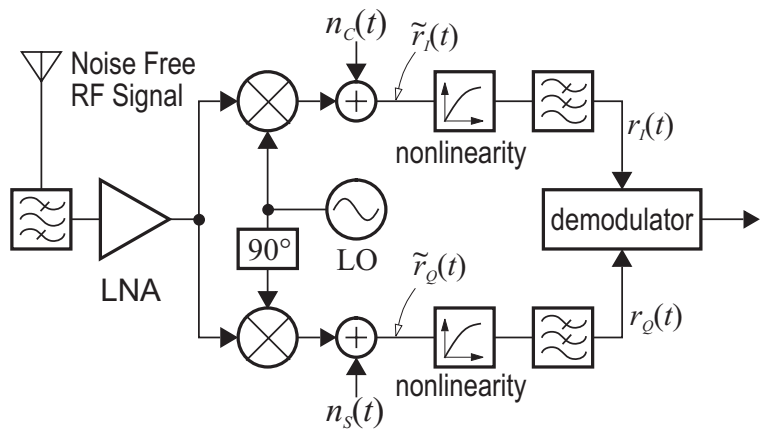

Fig. 1. The receiver model. The demodulator is shown in Fig. 2.

A high level model of the direct conversion receiver working in the AWGN channel is shown in Fig. 1. We first assume the RF section is ideal so that the noise free signal can be directly down converted to the base-band in-phase and quadrature-phase components without distortion. The corresponding base-band Gaussian noise terms are added in at the output of the mixer pair. Each of the I and Q signals is then passed to a polynomial block to model the distortion introduced by the RF front-end. Finally the channel selection is done by an 11th order Chebyshev filter pair. Neglecting the high frequency components, at the input of the polynomial block, we have

$$
\begin{aligned}
& \tilde{r}_{I}(t)=A \cos \phi(t)+n_{c}(t) \\
& \tilde{r}_{Q}(t)=A \sin \phi(t)+n_{s}(t)
\end{aligned}
$$

where $A$ is the signal amplitude; $n_{c}(t)$ and $n_{s}(t)$ are the equivalent base-band components of the band-limited input noise and

$$
\phi(t)=2 \pi f_{d} \int_{0}^{t} \sum_{n=0}^{\infty} a_{n} g(\tau-n T) d \tau
$$

is the signal phase introduced by the frequency modulation. In equation (2) $f_{d}=3.2 \mathrm{kHz}$ is the FSK tone spacing; $a_{n} \in$ 
$\{ \pm 1, \pm 3\}$ is the 4 -ary symbol value and $g(t)$ is a rectangular pulse of unit amplitude and duration $T$, the symbol period. These signals are amplified and distorted by the polynomial block which has the function of

$$
y(t)=a_{1} x(t)+a_{2} x^{2}(t)+a_{3} x^{3}(t)
$$

where $x(t)$ and $y(t)$ are the input and output signals respectively; $a_{1}, a_{2}$ and $a_{3}$ are constants. The second and third order input intercept points of the front-end are approximated by

$$
\begin{aligned}
& I I P 2 \approx \frac{a_{1}}{a_{2}} \\
& I I P 3 \approx \sqrt{\frac{4}{3}\left|\frac{a_{1}}{a_{3}}\right|}
\end{aligned}
$$

in linear scale. When referred to the $50 \Omega$ resistance, their more frequently used $\mathrm{dBm}$ equivalents are

$$
\begin{aligned}
& I I P 2=10 \log \left(10 \frac{a_{1}^{2}}{a_{2}^{2}}\right) \\
& I I P 3=10 \log \left\{10 \times \frac{4}{3}\left|\frac{a_{1}}{a_{3}}\right|\right\}
\end{aligned}
$$

\section{B. The Demodulator Model}

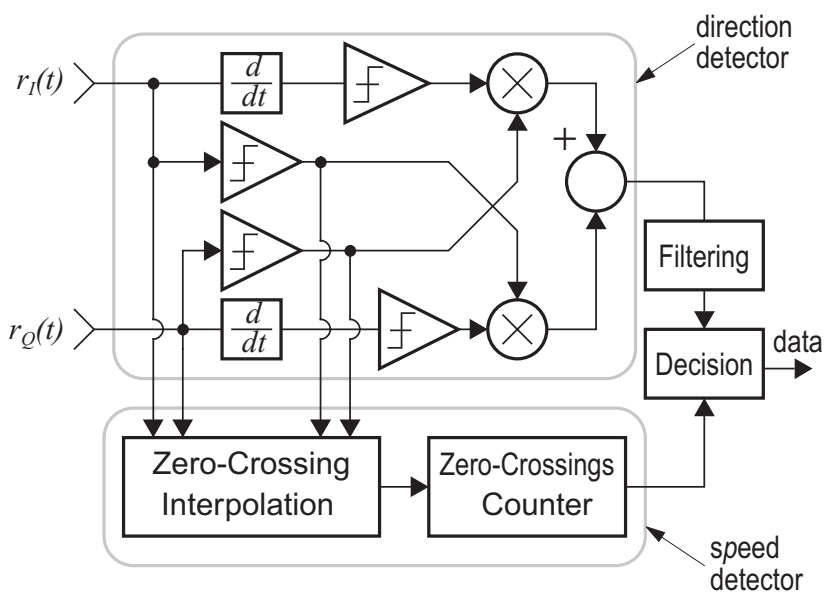

Fig. 2. The demodulator used in the receiver of Fig. 1. It is capable of detecting the 4-FSK FLEX signal. The zero-crossing interpolation technique is utilized to improve the detection accuracy.

The demodulator model is shown in Fig. 2. In a phasor diagram, the 4-FSK signal can be characterized by the direction and speed of the phasor as it rotates. The demodulator accordingly consists of a direction detector and a speed detector. The phasor rotation direction is detected by observing the phase relationship between the I and Q signals, the same method that has been implemented in binary FSK receivers. The rotation speed is estimated by counting and comparing the number of zero-crossings produced by the I and Q signals during one symbol period. A decision block combines the direction and speed information to recover the original bits. The ZCI technique helps generate more zero-crossings by means of addition and subtraction of $r_{I}(t)$ and $r_{Q}(t)$ and consequently relax the decision threshold and improve the detection accuracy.

\section{Official Specifications}

The HKOFTA has defined 5 parameters for VHF public radio paging receivers as the minimum performance requirements [5]:

1. reference sensitivity,

2. spurious response rejection,

3. desensitization,

4. intermodulation response rejection, and

5. radiated spurious emissions.

These are originally defined for the POCSAG system. However, as a standard for paging service, they should apply equally to the new system. As specifications 2) and 5) are more circuit-level related issues and can be satisfied with careful circuit design and layout, we include only the other three in this high level simulation.

By definition, the reference sensitivity is the field strength surrounding the receiver antenna and is $3 \mathrm{~dB}$ above the minimum value required for a successful calling rate of $80 \%$. The equivalent bit error rate (BER) for the $80 \%$ successful calling rate is $3 \%$ in the POCSAG system[6]. In our simulation we used $1 \%$ BER as the borderline and defined the sensitivity as the minimum input signal power to the LNA to produce this BER. Therefore in this paper, the reference sensitivity and the sensitivity are two related terms with different meanings. Given the field strength to determine the amount of power available for the LNA we need to know the efficiency and the effective aperture of the antenna as well as the carrier frequency[7]. The conversion problem will be addressed in next section.

To measure the desensitization, an un-modulated signal which is at least one channel away from the nominal operating frequency is introduced as the interference. The desired signal is adjusted to yield a field strength that is equivalent to the reference sensitivity level around the antenna. The desensitization is the difference between the interference and the wanted signal in $\mathrm{dB}$ when the successful calling rate is reduced by the interferer to $80 \%$. In the simulation the interferer is exactly one channel away from the desired signal.

The intermodulation response rejection $(I M R R)$ measurement is a little more complicated. According to the HKOFTA specification, a testing signal at the nominal operating frequency $f_{0}$ is first applied to yield a successful call ing rate of $80 \%$. Its level $U$ is recorded. The signal frequency is then changed to $f_{0}+2 n f_{c h}$ where $n= \pm 4$ and $f_{c h}$ is the channel spacing. At the same time, an unmodulated carrier at $f_{0}+n f_{c h}$ is introduced. The levels of these two signals are kept the same and increased to a minimum value $V$ at which a false decoder response is obtained. The $I M R R$ is $V-U$ in dB. An alternative definition is formulated by the Ministry of Post and Telecommunications of China[8]. It also uses the signal level $U$ as the reference. But unlike the HKOFTA specification, the level of the first testing signal is increased by $3 \mathrm{~dB}$ from $U$ while its frequency is unchanged. Two equal level unwanted signals are then applied. One of them is an un-modulated carrier at $f_{0}+m f_{c h}(m= \pm 1, \pm 2$ or \pm 4$)$ and the other is an FM signal at $f_{0}+2 m f_{c h}$. The FM signal is modulated 
by a $400 \mathrm{~Hz}$ sinusoid with a peak frequency offset of $3 \mathrm{kHz}$. The level of these two signals are increased to $W$ at which the successful calling rate drops to $80 \%$. The $I M R R$ is $W-U$ in dB. Since the BER is not an intuitive indicator of the decoder response, we chose the second method for the $I M R R$ simulation.

\section{Simulation Results}

The simulation is carried out to verify the performance of the demodulator, especially the sensitivity and the interference rejection ability, as well as to investigate the circuit requirements on the $\mathrm{RF}$ front-end. It is done with an effort to follow the setup which is suggested in formal measurements. Besides the specifications described in section II, impacts of I/Q phase mismatch and LO frequency drift are also studied. The channel spacing is $25 \mathrm{kHz}$. Bandwidth of the channel selection filter is about $8 \mathrm{kHz}$.

\section{A. Sensitivity}

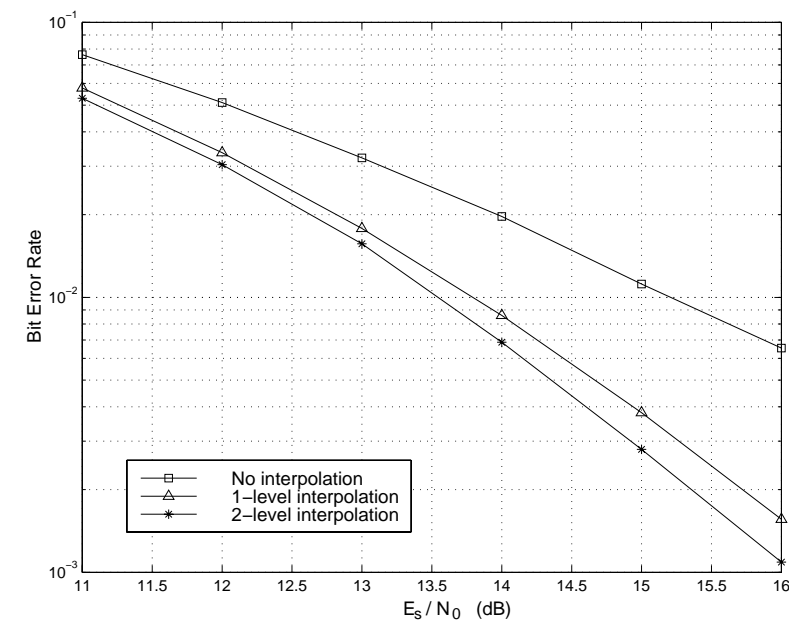

Fig. 3. The sensitivity simulation result at $-36 \mathrm{dBm}$ overall IIP3 and $11 \mathrm{dBm}$ IIP2. The $1 \%$ bit error rate is achieved with about 13.5 $\mathrm{dB} E_{S} / N_{0}$ ratio or $-125.3 \mathrm{dBm}$ input signal power when 2-level interpolation is applied.

As shown in Fig. 3, for the demodulator with 2-level interpolation, the $1 \% \mathrm{BER}$ level is achieved at an $E_{s} / N_{0}$ ratio of about $13.5 \mathrm{~dB}$ when the overall front-end $I I P 2$ and IIP3 are $11 \mathrm{dBm}$ and $-36 \mathrm{dBm}$, respectively. Since

$$
\frac{E_{s}}{N_{0}}=\frac{P s / R_{s}}{N_{0}}
$$

where $N_{0}=k T$ is the thermal noise spectral density; $P s$ is the signal power; $R_{s}=3200$ symbols per second is the baud rate, we have

$$
\begin{aligned}
P s & =10 \log \left(k T \cdot R_{s}\right)+\frac{E_{s}}{N_{0}}+30 \\
& \approx-125.3(\mathrm{dBm})
\end{aligned}
$$

at room temperature $\left(300^{\circ} \mathrm{K}\right)$. Assume the equivalent noise bandwidth $B$ is $9 \mathrm{kHz}$, the signal to noise ratio $(S N R)$ is:

$$
S N R=\frac{E_{s}}{N_{0}}+10 \log \left(\frac{R_{s}}{B}\right) \approx 9
$$

At the presence of interference components, as is the case in the desensitization and $I M R R$ testing, the signal to noiseand-interference ratio $(S N I R)$ should also be kept above this level in order to maintain the successful calling rate. Neglecting the noise contribution of the base-band stages, the receiver sensitivity is only limited by the noise performance of the RF front-end. To achieve $-120 \mathrm{dBm}$ sensitivity the front-end noise figure $(N F)$ must therefore be as low as $5.3 \mathrm{~dB}$. This number will be used in the following section to determine the RF front-end gain and noise budget. The maximum reference sensitivity required by the HKOFTA is $26 \mathrm{~dB} \mu \mathrm{V} / \mathrm{m}$. Using the same antenna parameters given by [7] and assuming perfect LNA input matching, this field strength is equal to $-109 \mathrm{dBm}$ signal power input to the LNA at $280 \mathrm{MHz}$ nominal operating frequency and the equivalent receiver sensitivity is $3 \mathrm{~dB}$ lower, i.e. $-112 \mathrm{dBm}$. Hence we have an $8 \mathrm{~dB}$ margin to make up for the errors resulted from our simple model.

It is noticeable that a continued increase in the level of interpolation will yield only diminishing improvement. The extra cost of hardware will probably not justify it.

\section{B. Desensitization}

The same method that is used in the HKOFTA desensitization testing is applied in the simulation. To provide the reference sensitivity, the $E_{s} / N_{0}$ ratio is set to $16.5 \mathrm{~dB}$. An un-modulated interference signal which is $65 \mathrm{~dB}$ higher than and one channel apart from the wanted one is introduced to verify the performance. Since the minimum desensitization requirement is $65 \mathrm{~dB}$, a BER of $1 \%$ or lower is expected. Simulation results at $25 \mathrm{~dB}$ gain $\left(a_{1}=17.8\right)$ are depicted in Fig. 4 and Fig. 5 in the format of BER versus $a_{2}(I I P 2)$ and $a_{3}(I I P 3)$ of the front-end, respectively. The relationship between the intercept points and the coefficients $a_{2}, a_{3}$ is given in (5). Not surprisingly, the 2 nd order distortion plays a critical role because of the resultant low frequency components which fall into the baseband and therefore can not be filtered out. As a result, the RF front-end must yield at least $11 \mathrm{dBm} \mathrm{IIP2}$ to satisfy the desensitization requirement.

Fig. 5 is plotted here as a comparison. One shouldn't come to the conclusion that the receiver is able to work at very poor $I I P 3$ s. In fact, as the only interferer in this simulation is an un-modulated carrier which is one channel away from the nominal, the 3rd order distortion creates no in-band interference at all. This is different from the case of intermodulation response.

\section{Intermodulation Response Rejection}

As described in part $\mathrm{C}$ of last section, two unwanted signals are present at $f_{0}+m f_{c h}$ and $f_{0}+2 m f_{c h}$ respectively, where $f_{0}$ and $f_{c h}$ are the nominal frequency and the channel spacing; $m$ could be plus or minus 1,2 , or 4 . Fig. 6 shows that the $I M R R$ is about $58 \mathrm{~dB}, 3 \mathrm{~dB}$ higher than the 


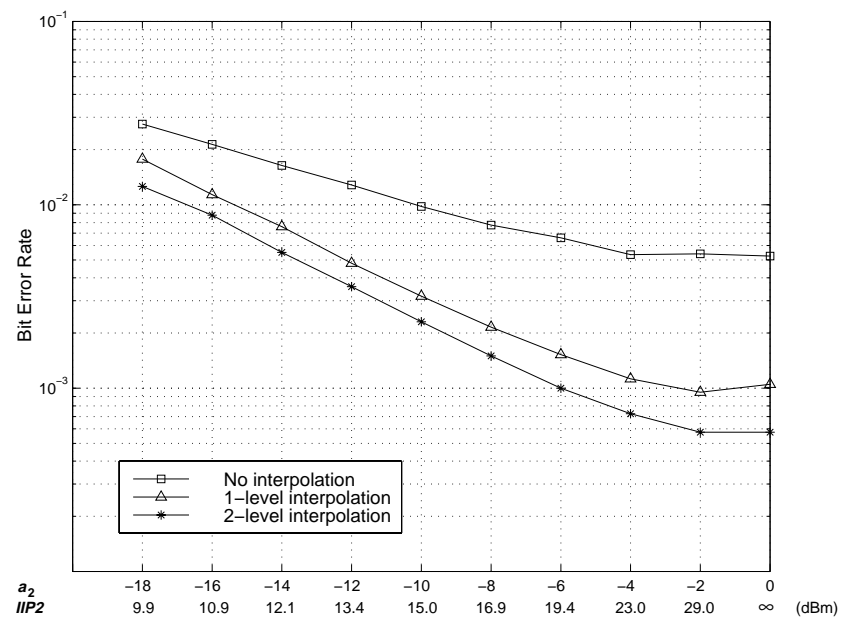

Fig. 4. Desensitization affected by the 2nd order nonlinearity. The interference is one channel apart. The x-axis shows both the coefficient $a_{2}$ and its corresponding $I I P 2$ values. $25 \mathrm{~dB}$ gain, -36 $\mathrm{dBm} I I P 3$ and 2-level interpolation.

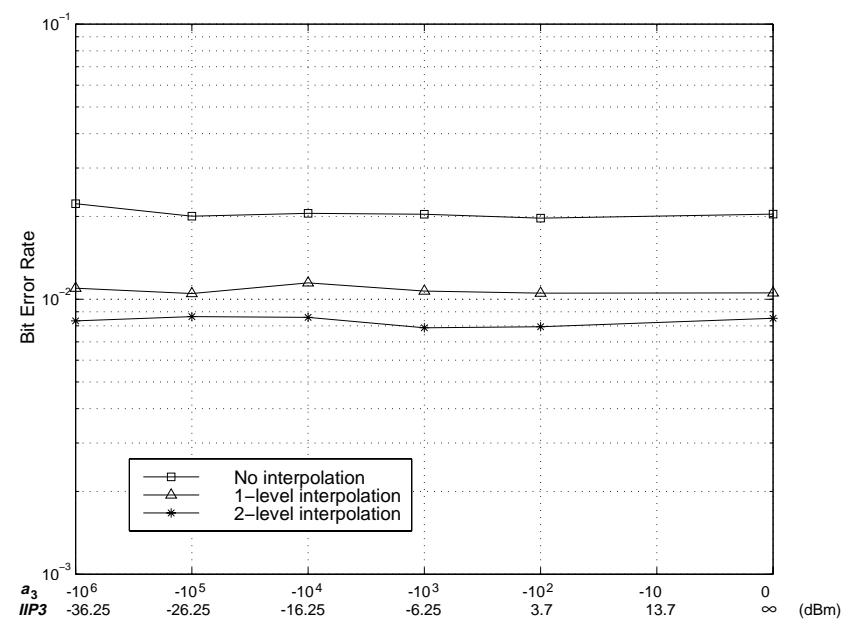

Fig. 5. Desensitization affected by the 3rd order nonlinearity. The $\mathrm{x}$-axis shows both the coefficient $a_{3}$ and its corresponding IIP3 values. $25 \mathrm{~dB}$ gain, $11 \mathrm{dBm}$ IIP2 and 2-level interpolation.

specification, with $m= \pm 1$. Better results are obtained when the unwanted signals get farther.

The $I M R R$ specification can be use to estimate the $S F D R$ (spurious free dynamic range) and required IIP3. As we already set the sensitivity to be $-120 \mathrm{dBm}$, the 55 $\mathrm{dB} I M R R$ implies $55+(-120)=-65 \mathrm{dBm}$ interference level. If the equivalent noise bandwidth $B$ is restricted to $9 \mathrm{kHz}$, with $5.3 \mathrm{~dB} N F$ the input referred noise floor is

$$
\begin{aligned}
N_{\text {floor }} & =10 \log (k T B)+N F+30 \\
& \approx-129(\mathrm{dBm})
\end{aligned}
$$

The $S F D R$ can be calculated by

$$
S F D R=-65-N_{\text {floor }} \approx 64 \quad(\mathrm{~dB})
$$

Finally the $I I P 3$ required to achieve this $S F D R$ is

$$
I I P 3=\frac{3}{2} S F D R+N_{\text {floor }} \approx-33 \quad(\mathrm{dBm})
$$

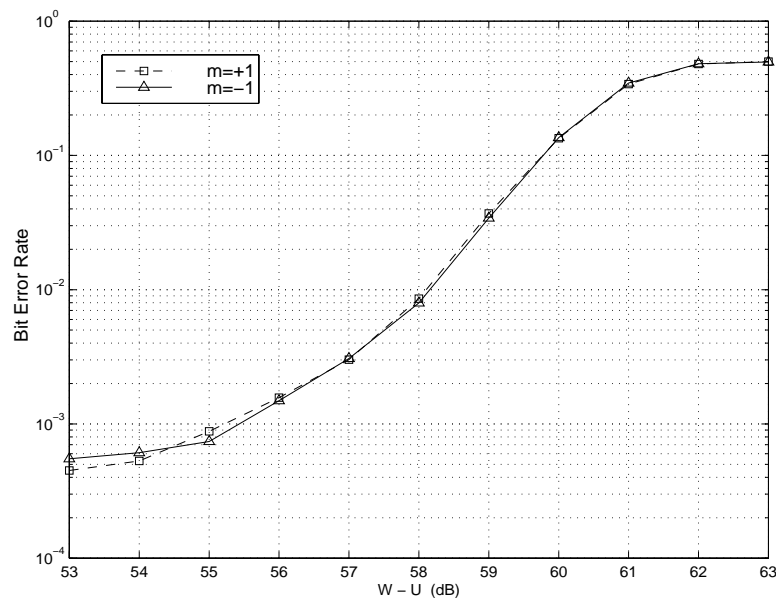

Fig. 6. The $I M R R$ is $58 \mathrm{~dB}$ when the unwanted signals are 1 and 2 channels above $(m=1)$ or below $(m=-1)$ the nominal, respectively. The meanings of $m, W$ and $U$ are defined in part $\mathrm{C}$ of section II.

The result is close to the $-36 \mathrm{dBm}$ value used in the simulation.

\section{Phase Mismatch, Frequency Drift and Phase Noise}

It turns out that the demodulator can withstand quite a large phase mismatch between the I and Q signals. A $10^{\circ}$ mismatch results in only about $0.1 \mathrm{~dB}$ degradation in the 12 to $16 \mathrm{~dB} E_{s} / N_{0}$ range, as shown in Fig. 7. Modern IC technology can reduce the mismatch to a much lower level. At the same time, severe performance degradation is observed when the LO drifts from the nominal carrier frequency. In Fig. 8 a $300 \mathrm{~Hz}$ drift worsens the sensitivity by $0.25 \mathrm{~dB}$. At the $280 \mathrm{MHz}$ band, this drift frequency translates to about $1 \mathrm{ppm}$ frequency stability. Over the 15 to $35^{\circ} \mathrm{C}$ temperature range for normal condition test, this is very difficult, if not impossible, to achieve without careful (and probably expensive) compensation. It is therefore necessary to apply some kind of automatic frequency control (AFC) and/or adaptive demodulation schemes. An AFC algorithm proposed in [9] demonstrated the convergence of the drift frequency from 1 to within $1 / 10$ of the symbol rate (about $3 \mathrm{kHz}$ ). Another consideration is the receiver bandwidth. The $8 \mathrm{kHz}$ bandwidth is not supposed to accommodate the drift. In a practical application, however, some allowance must be provided to alleviate the problem. The bottom line is to maintain an acceptable sensitivity level. Phase noise, on the other hand, is usually not a problem in paging receivers where crystal oscillators are commonly used. Ultra low phase noise below $-100 \mathrm{dBc} / \mathrm{Hz}$ can be achieved at a few hundred hertz[10]. Reciprocal mixing at this level is completely negligible.

\section{RF Front-End Budget}

The sensitivity and desensitization simulation provides important information for the system planning. Once the overall noise and nonlinearity requirements have been determined, a budget can be given for the front-end including the LNA and the mixer. The $5.3 \mathrm{~dB}$ overall $N F$ which is 


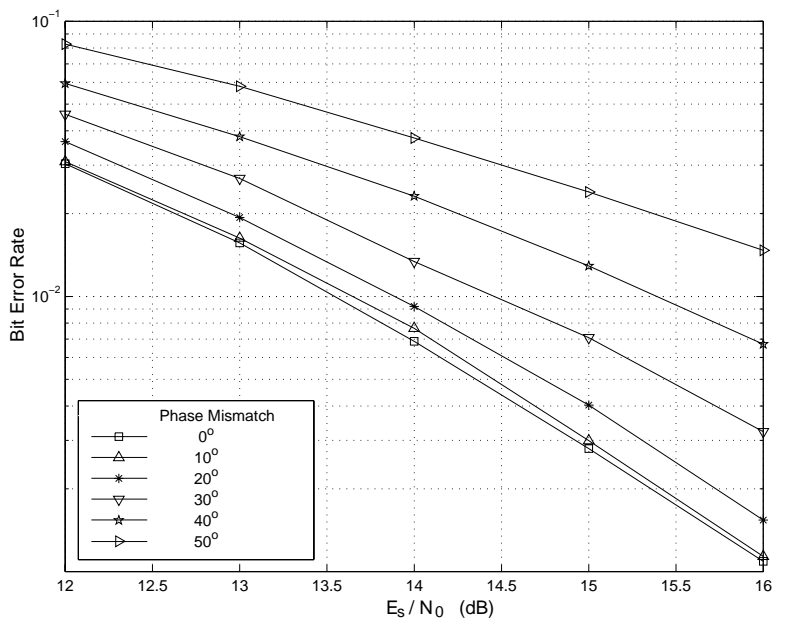

Fig. 7. Sensitivity degradation due to I/Q phase mismatch. A $10^{\circ}$ mismatch results in only about $0.1 \mathrm{~dB}$ degradation.

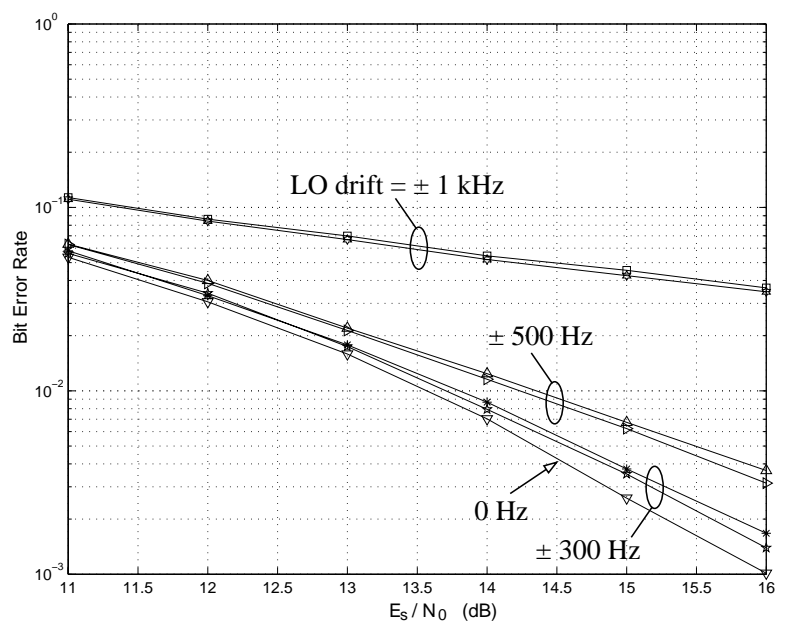

Fig. 8. Sensitivity degradation due to LO frequency drift. The LO frequency stability should be kept around $1 \mathrm{ppm}$ for a decent BER.

required to achieve the $-120 \mathrm{dBm}$ sensitivity is actually a very stringent number. The LNA must provide enough gain to suppress the noise contribution from the following stages. As an example, assume the LNA gain is $16 \mathrm{~dB}$ and the mixer $N F$ is $15 \mathrm{~dB}$, the LNA $N F$ is then given by

$$
\begin{aligned}
N F_{L N A} & =10 \log \left(F_{\text {total }}-\frac{F_{\text {mixer }}-1}{G_{L N A}}\right) \\
& \approx 4.2(\mathrm{~dB})
\end{aligned}
$$

Where $F_{\text {total }}$ and $F_{\text {mixer }}$ are the noise factors of the frontend and the mixer; $G_{L N A}$ is the LNA power gain in linear scale.

Although $4 \mathrm{~dB} N F$ may seem to be an easy objective, it must be justified with caution because the designers may want the LNA to be differential. In a heterodyne receiver the single-ended LNA is desired because of its superior noise performance. However, for a direct conversion receiver like the one under discussion, the differential architecture may be more appropriate for its excellent cancella- tion of the 2 nd order distortion. In the case of low source impedance, the equivalent input noise voltage of a differential pair is $3 \mathrm{~dB}$ higher than a single common emitter amplifier when their transistors are biased at the same current[11]. The doubled equivalent input noise power won't push the $N F$ by the same amount but its contribution is significant. A straightforward calculation shows 1.4 to 1.8 $\mathrm{dB}$ increase in the $N F$ when the original single-ended value is somewhere between 2 and $3 \mathrm{~dB}$. Taking other important factors like the nonlinearity, the input matching and the power consumption into account, the LNA gain and noise budget is still feasible but may require extra efforts. We then assign $9 \mathrm{~dB}$ gain to the mixer so that an overall gain of $25 \mathrm{~dB}$ could be obtained from the RF section. The 9 $\mathrm{dB}$ assignment is somewhat arbitrary and may include a buffer stage.

Having done the gain and $N F$ budget, we can determine the nonlinearity distribution by using the polynomial approximation. Constant overall IIP2 and IIP3 curves of the front-end are given in Fig. 9 and Fig. 10. The $\mathrm{x}$ and $\mathrm{y}$ variables are the intercept points of the LNA and the mixer, respectively. With typical LNA and mixer IP values, the system nonlinearity requirements should be satisfied without any difficulty. Table I summarizes the planning. Some design margin has been deliberately made available.

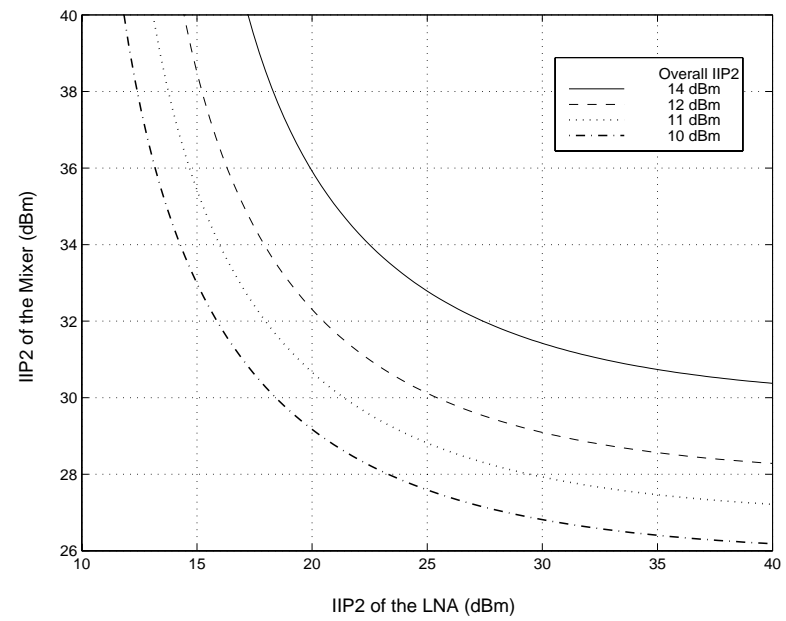

Fig. 9. Constant overall IIP2 curves. A combination of $25 \mathrm{dBm}$ LNA $I I P 2$ and $30 \mathrm{dBm}$ mixer IIP2 yields $12 \mathrm{dBm}$ cascaded IIP2.

TABLE I

RF Front-End Budget.

\begin{tabular}{l||l|l|l}
\hline & LNA & Mixer & Overall \\
\hline \hline Gain $(\mathrm{dB})$ & 16 & 9 & 25 \\
\hline NF $(\mathrm{dB})$ & 4 & 15 & 5.2 \\
\hline IIP2 $(\mathrm{dBm})$ & $\geq 25$ & $\geq 30$ & $\geq 12$ \\
\hline IIP3 $(\mathrm{dBm})$ & $\geq-26$ & $\geq-12$ & $\geq-30$ \\
\hline
\end{tabular}




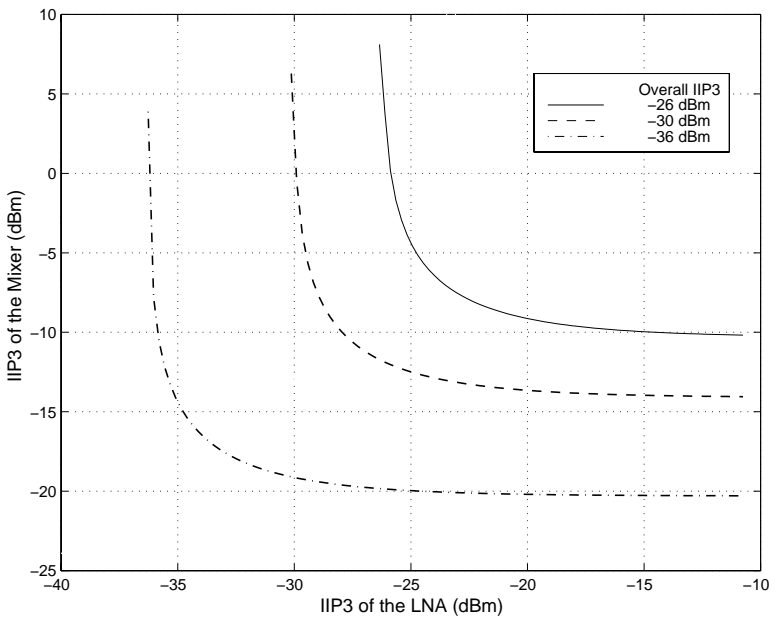

Fig. 10. Constant overall IIP3 curves. An overall IIP3 of $-30 \mathrm{dBm}$ can be achieved with $-26 \mathrm{dBm}$ LNA $I I P 3$ and $-12 \mathrm{dBm}$ mixer IIP3.

\section{Conclusion}

The simulation results and RF front-end budget of a zero-crossing interpolation based direct conversion FLEX paging receiver were presented. Receiver criteria including the sensitivity, the desensitization, I/Q phase mismatch and LO frequency drift are simulated. With a reasonably good RF front-end, the official sensitivity and desensitiza tion specifications are satisfied.

\section{REFERENCES}

[1] I.A.W. Vance, "Fully Integrated Radio Paging Receiver," IEE Proc., Vol. 129, Pt. F, No. 1, pp. 2-6, Feb. 1982.

[2] J. F. Wilson, R. Youell, T. H. Richards, G. Luff, and R. Pilaski, "A Single-Chip VHF and UHF Receiver for Radio Paging," IEEE J. Solid-State Circuits, Vol. 26, No. 12, pp. 1944-1950, Dec. 1991.

[3] Z. Chen, J. Lau, "Direct Conversion for FLEX Paging Receivers - A Feasibility Study," 1998 IEEE RFIC Symp. Dig., Baltimore, MD, pp. 293-296.

[4] H. M. Kwon and K. B. Lee "A Novel Digital FM Receiver for Mobile and Personal Communications," IEEE Trans. Commun., Vol. 44, No. 11, pp. 1466-76, Nov. 1996.

[5] OFTA Hong Kong, Performance Specification for VHF Transmitters and Receivers for Use in The Public Paging Service, HKTA 1004, Issue 3, Jul. 1993. URL: http://www.ofta.gov.hk/standards/hktaspec/taspec.html.

[6] Philips Semiconductors, Pager Application Handbook, 1995, pp.VI-41 to 42 .

[7] T.L. Fu, "Optimize The Performance of Pager Antennas," Microwaves \& RF, Aug. 1994, pp. 141-147.

[8] The Ministry of Post and Telecommunications of China, Specifications and Their Testing Methods of Radio Paging Receivers, Beijing: The Standards' Publishing House of China, Aug. 1998.

[9] M. Saitou, M. Kawabata and Y. Akaiwa, "Direct Conversion Receiver for 2- and 4-level FSK Signals," 1995 4th IEEE Int. Conf. on Universal Personal Communications. New York, NY, pp. 392-6.

[10] Q. Huang and P. Basedau, "Design Considerations for HighFrequency Crystal Oscillators Digitally Trimmable to Sub-ppm Accuracy," IEEE Trans. Very Large Scale Integration (VLSI) Syst., Vol. 5, No. 4, pp. 408-416, Dec. 1997.

[11] P. Gray and R. Meyer, Analysis and Design of Analog Integrated Circuits, 3rd edition, New York: Wiley, 1993, Chapter 11.
Zhiheng Chen (S'98) received his BEng and MEng degrees in radio engineering from Southeast University, Nanjing, China, in 1992 and 1995 , respectively. He is currently working toward the $\mathrm{PhD}$ degree in electrical and electronic engineering at the Hong Kong University of Science and Technology. His research interests include analog and RF IC design, low noise techniques and radio receiver architectures.

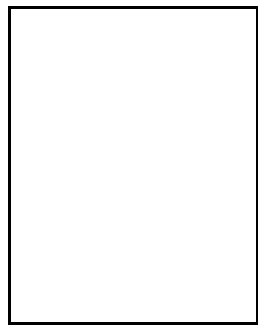

Jack Lau (S'89-M'90) obtained his BS and MS degrees in Electrical Engineering from the University of California at Berkeley under the Honors Degree Program (Option H). He completed his Ph.D. degree in 1994 and became the first Ph.D. to graduate from the Hong Kong University of Science and Technology. His MS thesis was on the design and analysis of integrated pin drivers for in-circuit testing. While at Berkeley, he worked at Hewlett Packard as a technical support intern and at Schlumberger as an automatic test equipment engineer. After obtaining his MS degree, he worked as a CPU design engineer at the Integrated Information Technology Santa Clara, California. His Ph.D. thesis was on integrated magnetic sensors in both bulk silicon and SOI technologies. From 1995-96, he was a Visiting Scholar at Center for Integrated Systems at Stanford University researching in RF CMOS circuits and substrate noise coupling issues.

Jack Lau is currently an Assistant Professor at the Department of Electrical and Electronic Engineering and a Director of the Consumer Media Laboratory at the Hong Kong University of Science and Technology.

He is a recipient of the UC Berkeley Alumni Scholarship, Cray Research Scholarship, Unisys Scholarship, and other awards. In 1995, he received the 1995 School of Engineering Faculty Teaching Appreciation Award. He is also a member of IEEE, Eta Kappa Nu, and Tau Beta Pi. 